\title{
Individual differences in social, cognitive, and morphological aspects of infant pointing
}

\author{
Ulf Liszkowski ${ }^{\mathrm{a}, \mathrm{b}, *}$, Michael Tomasello ${ }^{\mathrm{c}}$ \\ a Max Planck Research Group Communication Before Language, Max-Planck-Institute for Psycholinguistics, Nijmegen, The Netherlands \\ b Donders Institute for Brain, Cognition, and Behaviour, Radboud University, Nijmegen, The Netherlands \\ c Department of Developmental and Comparative Psychology, Max-Planck-Institute for Evolutionary Anthropology, Leipzig, Germany
}

\section{A R T I C L E I N F O}

\section{Keywords:}

Gesture

Pointing

Communication

Individual differences

Mother-child relations

Developmental origins

\begin{abstract}
A B S T R A C T
Little is known about the origins of the pointing gesture. We sought to gain insight into its emergence by investigating individual differences in the pointing of 12-month-old infants in two ways. First, we looked at differences in the communicative and interactional uses of pointing and asked how different hand shapes relate to point frequency, accompanying vocalizations, and mothers' pointing. Second, we looked at differences in social-cognitive skills of point comprehension and imitation and tested whether these were related to infants' own pointing. Infants' and mothers' spontaneous pointing correlated with one another, as did infants' point production and comprehension. In particular, infants' indexfinger pointing had a profile different from simple whole-hand pointing. It was more frequent, it was more often accompanied by vocalizations, and it correlated more strongly with comprehension of pointing (especially to occluded referents). We conclude that whole-hand and index-finger pointing differ qualitatively and suggest that it is index-finger pointing that first embodies infants' understanding of communicative intentions.
\end{abstract}

(C) 2011 Elsevier Inc. All rights reserved.

The human pointing gesture is a species-unique, species-universal communicative device that paves the way for early language (Bates, Camaioni \& Volterra, 1975; Butterworth, 2003; Tomasello, Carpenter \& Liszkowski, 2007). It continues to play a pivotal role throughout language development, including the transition to syntax (Capirci, Iverson, Pizzuto, \& Volterra, 1996; Iverson \& GoldinMeadow, 2005). Surprisingly, very little is known about the ontogenesis of the pointing gesture.

\footnotetext{
* Corresponding author at: Max Planck Institute for Psycholinguistics, Wundtlaan 1, 6525 XD Nijmegen, The Netherlands.

E-mail address: ulf.liszkowski@mpi.nl (U. Liszkowski).
} 
Age of emergence of the pointing gesture ranges from 8 to 15 months (Carpenter, Nagell \& Tomasello, 1998; Leung \& Rheingold, 1981; Murphy \& Messer, 1977). Accounting for individual differences in this emergence may provide insight into the origins of pointing. One theory is that early pointing originates from attempts to reach for an object (Vygotsky, 1978). However, this account is not well supported. First, infants use reaching and pointing in different contexts (Franco \& Butterworth, 1996), and once pointing has emerged, reaching remains in children's repertory with equal frequency, suggesting that these two forms are developmentally and functionally unrelated (Masataka, 2003). Second, 12-month-olds point to indicate the whereabouts of sought-for objects (Liszkowski, Carpenter \& Tomasello, 2008), and to express and share interest in specific events (Camaioni, Perucchini, Bellagamba \& Colonnesi, 2004; Liszkowski, Carpenter \& Tomasello, 2007), both uses of pointing that could not plausibly emerge from requesting objects.

A second theory is that infants initially point non-communicatively for themselves, and later this behavior becomes communicative (Bates et al., 1975). This account is also not well supported. First, it is based mainly on the observation that infants sometimes do not coordinate their gaze with a potential recipient of the communication. However, for a variety of reasons, gaze alternation is not a valid or reliable indicator of communicative intent (Harris, Barlow-Brown \& Chasin, 1995; Liszkowski, Albrecht, Carpenter \& Tomasello, 2008; Moore \& Corkum, 1994). Second, beginning pointers point less when they are alone in a room than when a social partner is present (Franco, Perucchini, \& March, 2009), and infants continue to point non-communicatively even after they have begun pointing communicatively (Delgado, Gomez, \& Sarria, 2009), suggesting that communicative pointing emerges either earlier than or at the same time as non-communicative pointing.

In contrast to these individualistic accounts of early pointing (i.e. 'pointing-for-reaching' and 'pointing-for-self'), social-pragmatic accounts suggest that pointing is part of social activity from the beginning. Bruner (1983) emphasized that acts of reference originate from a primal motive to share experiences with social partners and are essentially collaborative acts. Similarly, Werner and Kaplan (1963) proposed that the emergence of reference is mediated by the social practice of "sharing contemplated objects in an interpersonal context" (p. 79). Tomasello et al. (2007) have argued and presented evidence that infant pointing is based on more general social-cognitive skills of shared intentionality, such as the ability to form joint intentions and joint attention with others in nonlinguistic activities.

These social-pragmatic approaches hold the promise of accounting for differences in early pointing better than individualistic accounts. In the present study, we take a social-pragmatic perspective on human communication and provide data relevant to the broad question of the psychological origins of the human pointing gesture. Our method is intensive study of individual differences in early pointing (at 12 months of age). We investigate how infants on the cusp of pointing differ from each other in terms of social-interactional, social-cognitive, and morphological aspects of the gesture. Since pointing is a universal developmental attainment (except for pathological cases of autism; Baron-Cohen et al., 1996), individual differences in its emergence are potentially informative regarding the correlates and (by inference) the causes of developmental change (Bates, Dale \& Thal, 1995). Although correlations never by themselves establish direction of causality, establishing whether such correlations exist is an important first step in understanding the ontogenesis of human pointing.

In a first set of analyses, we investigated how mothers and infants point for one another. We looked for evidence of social-interactional usage by correlating infants' pointing with that of their mothers in a standardized observational setting. We tested the hypothesis that caregivers and infants use pointing as a shared practice of looking at things together. We designed a new point elicitation procedure involving a decorated room which parents were invited to look around with their infant. In line with our hypothesis, our first question was whether parents and infants would spontaneously use the pointing gesture while looking at the decorative items. Previous researchers have typically looked at either free play interactions with proximal toys, eliciting little pointing (Rheingold, Hay, \& West, 1976), or a mix of distal and proximal stimuli including picture books, which are specific to sociocultural reading practices (Lock, Young, Service, \& Chandler, 1990). Second, we assessed whether parents' spontaneous pointing was related to infants' pointing. Previous studies have used the caregiver as preprogrammed partner (Leung \& Rheingold, 1981); hence, we know little about caregivers' spontaneous input. Finally, we examined whether systematic differences exist in the morphology of infants' pointing gestures and whether these relate differently to their own vocalizations and their parents' pointing, possibly 
indicating different forms of communicative usage. The integration of manual pointing with the vocal modality provides a further cue regarding the communicative function of pointing and its later integration with speech. Previous studies have investigated point + word combinations (Capirci et al., 1996; Iverson \& Goldin-Meadow, 2005), but little is known about the vocalizations accompanying different morphological forms of pointing.

In a second set of analyses, we correlated infants' pointing behavior with experimental assessments of several different social-cognitive skills, including imitation and point comprehension. Here we wanted to know if infants' early pointing is correlated with understanding of the behavioral and communicative intentions of others. Specifically, we assessed different levels of point comprehension in three pointing tests, requiring infants to follow a point to a visible target (Carpenter et al., 1998), follow a point to an occluded target behind a barrier (Moll \& Tomasello, 2004), and infer the referential intent behind a point to a container where an object was hidden (Behne, Carpenter, \& Tomasello, 2005). Previous studies have looked at more global measures of production and comprehension but not at distal pointing per se (Carpenter et al., 1998; Mundy et al., 2007), or have correlated pointing with point-following to a visible target (Lempers, 1979; Leung \& Rheingold, 1981; Murphy \& Messer, 1977) but not with skills for inferring referential intentions as required when following a point to an occluded referent. We also investigated whether the various forms of point comprehension require the same unifying understanding of referential intentions or whether simple point following involves lesser cognitive skill than inferring others' referential intentions (Aureli, Perucchini \& Genco, 2009; but see commentary by Liebal \& Tomasello, 2009). We predicted joint emergence, indicated by correlations of point comprehension and production, especially for infants who pointed, like adults, with the index finger.

We also assessed several aspects of imitation, in particular infants' imitation of action goals and action styles (Meltzoff, 1995), their reproduction of manual gestures without objects (Uzgiris \& Hunt, 1975), and their ability to imitate and reverse roles (Carpenter, Striano, \& Tomasello, 2005). On the hypothesis that infants' pointing requires an understanding of others' actions and the ability to reproduce these, reproduction of action goals and styles should be related to pointing. In particular, reproducing action styles and gestures without objects might relate to the emerging form of the extended index finger, because this form has been suggested not to be culturally universal and so possibly is learnt by imitation (Wilkins, 2003). Further, based on the assumption that pointing involves an understanding of both roles, comprehension and production, the ability to reverse roles in an imitation game should be related to point production. Finally, to control for the specificity of the test relations we also administered a non-social, object permanence test which has been shown not to be associated with the emergence of joint attention (Carpenter et al., 1998).

In both sets of analyses, we were careful to distinguish so-called "whole-hand" pointing (Franco \& Butterworth, 1996; Lock et al., 1990) from index-finger pointing. Our question was whether these different morphological forms of indicative gesturing are functionally equivalent. If so, they should reveal similar correlational patterns in both sets of analyses. Alternatively, the different hand shapes could reflect different stages in development, or different gestures adapted for different social ends, and so reveal different associations with social-interactional and social-cognitive skills.

\section{Method}

\subsection{Participants}

Thirty-nine 12-month-olds (20 girls; mean age 366 days, range 349-398 days) were recruited from a database of mothers who had agreed to participate in infant studies. Mothers were intentionally left unaware that the study was about pointing. (They were told we were interested in infant development generally.) Eighty percent of mothers were at home full-time. Forty percent had an academic degree beyond high school. Eighty-two percent of the infants lived with both parents; the rest lived with their mothers. Fifty-six percent were first-borns. 

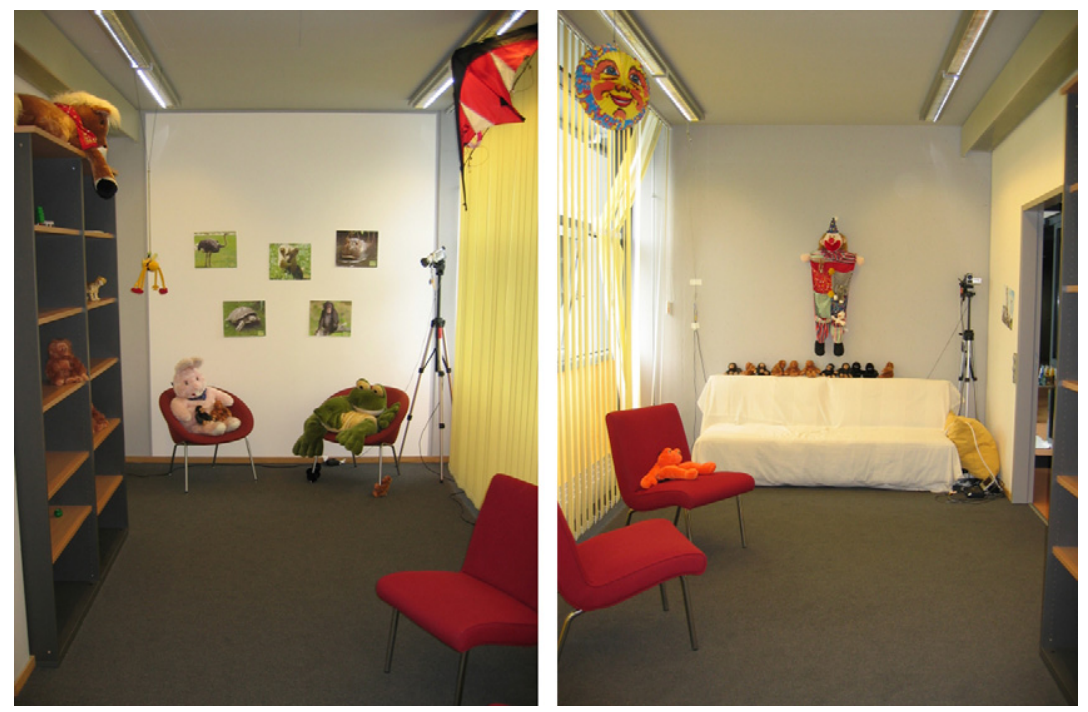

Fig. 1. Photo of the 'decorated room'.

\subsection{Setting}

Fig. 1 shows the 'decorated room' in which a number of interesting objects and pictures were displayed, broadly analogously to an exhibit or museum. These included, for example, different animal posters on the walls, a kite and colored paper lantern hanging from the ceiling, and shelves displaying various toys and dolls (e.g., blocks and teddy bears) of various sizes and colors. Four cameras recorded the scene from the four corners of the room through a quad-splitter on a mini-DV player positioned outside the room.

For the various assessments, mother, infant, and two experimenters sat on a rug in the middle of another room. On each of two opposite walls hung a small picture. A $1 \mathrm{~m} \times 0.8 \mathrm{~m}$ barrier stood perpendicular against another wall. Four cameras recorded the scene from each side.

\subsection{Procedure}

In the decorated room assessment, mothers were asked to hold their infant on their hip and take a look together at the things exhibited in the room. Pointing was never mentioned during the instructions. Mothers were asked to leave the objects in their places if possible and simply walk around in the room to look at the things together with their infant. The experimenter left mothers and infants alone in the room and recorded them for $5 \mathrm{~min}$.

Mothers and infants were then led to the other room for the series of assessments. These included three point comprehension tests, three imitation tests, and one object permanence test. They were administered in a fixed order within a natural play interaction which lasted about $30 \mathrm{~min}$. The order was chosen to be engaging and least tiring for infants: (1) object permanence, (2) simple point following, (3) goal-directed imitation (dumbbell test, bird test), (4) informative point comprehension, (5) role reversal imitation, (6) point following behind barrier, and (7) gesture imitation.

\subsubsection{Point comprehension}

Simple point-following (2 trials). Infants sat on their mother's lap facing the experimenter (E). E established eye contact, called the baby's name, and looked and pointed with the outstretched arm 
$90^{\circ}$ sideways to a $20 \mathrm{~cm} \times 20 \mathrm{~cm}$ picture on a wall $2 \mathrm{~m}$ away on the same side as the pointing arm. She said, "Oh, look! That's nice!" E then looked back to the infant and waited few seconds in case of a delayed response (see Carpenter et al., 1998). The sequence was repeated with E pointing to a picture on the opposite wall.

Informative point comprehension ( 2 trials). The apparatus consisted of a toy train and tracks that were attached to a $1 \mathrm{~m} \times 1 \mathrm{~m}$ square board on the floor. During an initial demonstration, infants and a second experimenter (E2) let the train roll down a bridge, pass a switch, and then go either to the left or the right and come to stop in front of E. Infants were encouraged to retrieve the train to repeat the game (approximate distance was $70 \mathrm{~cm}$ ). Then, the endpoints of the train on the left and right were covered with a cardboard roof, so that only the path of the train (but not its endpoint) remained visible. This procedure served to familiarize infants with finding the train beneath the roof. For the test, a tunnel occluded the switch and the rest of the tracks so that infants could not see which direction the train took. A noise (lasting approx. $3 \mathrm{~s}$ ) that a toy emitted when being activated by E overshadowed potential acoustic directional cues, as confirmed in pilot testing with 6 adults. E indicated the location of the train by pointing across the body to the train's occluded endpoint, alternating gaze between the infant and the location, repeatedly saying "There!" and "Look!" The cueing period lasted for approximately 20 s. If the infant did not retrieve the train, E gave it to the infant. The test trial was then repeated with the train rolling to the other side's endpoint.

Point-following behind a barrier ( 2 trials): Infants sat on their mother's lap facing E. Perpendicular to a middle line between the infant and $\mathrm{E}$, at a distance of approximately $1 \mathrm{~m}$, was a $1 \mathrm{~m} \times 1 \mathrm{~m}$ barrier. On E's side a toy was hidden behind the barrier. E's pointing and speech was the same as in the simple point-following test. To see the target infants had to crawl around the barrier (approximately $1 \mathrm{~m}$; see Moll \& Tomasello, 2004).

\subsubsection{Imitation}

Object-directed imitation ( 2 tests, each 2 trials). In the dumbbell test (Meltzoff, 1995), E gave infants a plastic dumbbell (approx. $15 \mathrm{~cm}$ long). After a 20-s baseline period, E demonstrated that a cap could be pulled off the end of the dumbbell. She held the dumbbell upright on the floor and, after calling the infant's attention, used a specific action-style by slowly extending her arm vertically into the air and then letting it sink down from above to pull off the cap. Then the dumbbell was given to the infant for a 30-s response period, E saying "Now you!" In the bird test, infants were first given a toy bird and a $60 \mathrm{~cm} \times 60 \mathrm{~cm}$ board on which a $10 \mathrm{~cm}$ square cardboard box was attached. After a 20-s baseline period, E used a specific action-style in making the bird hop up and down toward the box and then put it inside. Then infants were given the bird for a 30-s period, E saying "Now you!"

Gesture imitation (2 trials). Infants sat facing E. E called the infant's attention, established eye contact, raised her arms up and then let them fall down on her thighs. She repeated this twice looking expectantly at the infant for about $10 \mathrm{~s}$. If the infant showed no signs of copying the movement, E2 joined and imitated $\mathrm{E}$ in response to her demonstration. $\mathrm{E}$ then looked expectantly at the infant and encouraged her to imitate, saying "Now you!", again for about $10 \mathrm{~s}$. The second trial proceeded as the first, except that $\mathrm{E}$ clapped with her palm against her chest.

Role reversal (2 trials). This test was based on that of Carpenter et al. (2005, Study 2b). E sat in front of the infant and held a toy boat on her palm. With the other hand she placed a plastic toy tiger into the boat. Then she gave the toy tiger to the infant and encouraged the infant to place it into the boat, saying "Now you!" Then roles were reversed; E held the toy in her hand and gave the boat to the infant. The infant's task was now to hold the boat out so that E could put the toy tiger in.

\subsubsection{Object permanence (2 trials)}

In each of two trials, E showed the infant a small toy (a plastic dinosaur), verbally labeled it, and then hid it under one of the two cloths. After either E or the infant had retrieved it, E put it into a cup and transferred it invisibly from the cup to under one of the two cloths (invisible displacement; Uzgiris \& Hunt, 1975). E then asked, "Where is the Dino?" and repeated the question up to three times for a total period of $20 \mathrm{~s}$. 


\subsection{Coding}

Videotapes of mothers and infants in the decorated room were coded in both real-time and frame-by-frame analyses for the occurrence of pointing and vocalizing. Reliability was assessed by an independent coder who coded a random $25 \%$ of the sample. Pointing was coded based on the behavioral appearance of the arm either fully or half extended toward a discernable object or location, accompanied by looks in that direction (either during or shortly before or after the arm extension), and not a clear attempt to only grab or touch something or simply stemmed from lose arm movements or undirected excitement. Coders agreed on $96 \%$ and $91 \%$ of cases for mothers and infants, respectively, and number of points was highly correlated (respectively, Spearman's $\rho=.988, .986$ ). The hand shape of pointing was further differentiated into index-finger, when the index finger was clearly extended relative to all other fingers, and hand when not (Cohen's Kappa $=.82$ ). For infants, point accompanying vocalizing was coded if the vocalization was not fussing or otherwise non-communicative (e.g., hiccups, burps). In addition, the proto-demonstrative utterance "Da" ("There") was coded, yielding a Cohen's Kappa $=.77$.

Point accompanying gaze alternations between participants, as often reported in studies of infant gestural communication, were not coded. The nature of the activity (walking around freely) made it impossible to reliably record gaze alternations. Moreover, since infants were carried on mothers' hips, the aligned body orientations rendered the recipient's availability obvious, making it unnecessary for either infants or mothers to check each others' attention.

For the assessments conducted in the second room, each trial was coded separately.

\subsubsection{Point comprehension}

Simple point following. A pass was coded when infants turned their head sideways and looked at the picture. Cohen's Kappa $=.75$. Looks at the extended arm or index finger were not coded as point following. Informative point comprehension. A pass was coded when infants crawled toward the correct side and removed the lid to take or look at the train. Cohen's Kappa $=1$. Point following behind a barrier. A pass was coded when infants crawled behind the barrier to take or look at the toy. Cohen's Kappa $=1$.

\subsubsection{Imitation}

Object-directed imitation. A pass for copying the action goal was coded when infants pulled off the cap of the dumbbell or put the bird into the box. A pass for copying the action style was coded when infants held the dumbbell vertically or pulled off the cap from above or hopped the bird around. Cohen's Kappas for the dumbbell and bird tests respectively were .89 and .91 . Gesture imitation. A pass was coded if infants executed arm movements that were in response to and resembled the modeled gesture. Cohen's Kappa $=1$. Role reversal imitation. A pass was coded if infants reversed roles by holding out the boat so E could place the tiger into it. Cohen's Kappa $=1$.

\subsubsection{Object permanence}

A pass was coded if infants removed the cloth under which the toy was hidden and searched for or took the toy. Cohen's Kappa $=1$.

\section{Results}

\subsection{Infants' pointing}

The decorated room activity elicited spontaneous pointing in all but two infants. The distribution of points ranged from 0 to 32 points with a median of 7 and a mean of 10.85 . Because it diverged significantly from a normal distribution (Kolmogorov-Smirnov, $p=.023$ ), we used non-parametric statistics. Subsequent analyses revealed differences and interrelations in infants' pointing forms, frequency, and accompanying vocalizations.

With regard to hand shape, 18 infants pointed only with the whole hand (hand pointers) and another 19 infants pointed both with the whole hand and at least once with the index finger (index-finger pointers). Fig. 2 shows that index-finger pointers pointed overall significantly more than hand pointers 


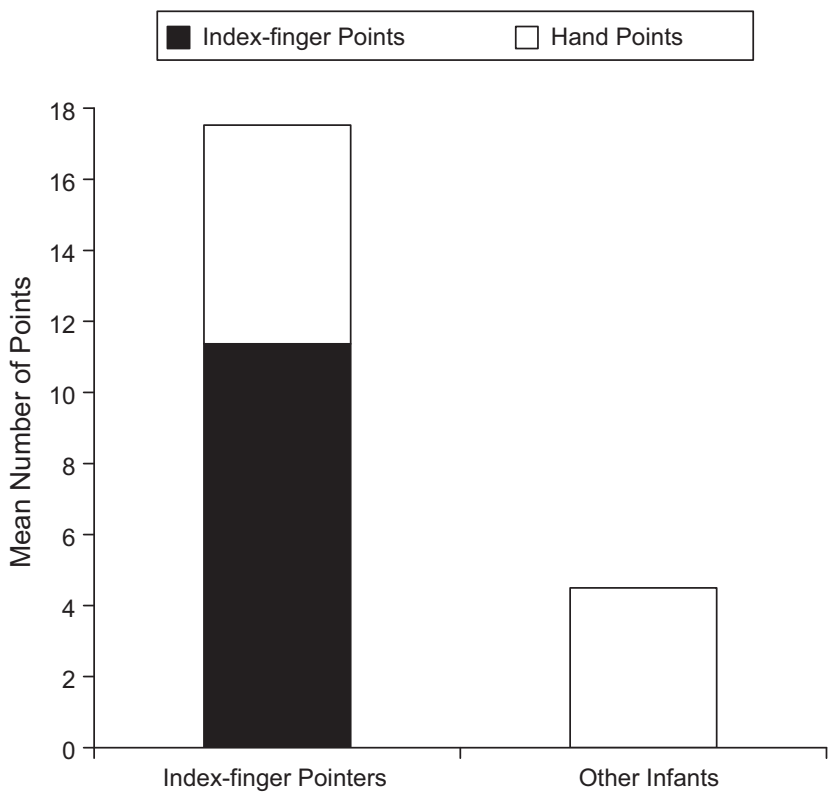

Fig. 2. Mean number of index-finger and whole-hand points for index-finger pointers and other infants.

(Mann-Whitney $U=26.0 ; p<.001$ ) and the number of whole-hand points did not differ significantly between hand pointers and index-finger pointers (Mann-Whitney $U=114 ; p=.204$ ).

Since index-finger pointers did not exclusively point with the index finger but also with the whole hand (mean proportion of index-finger points $=.61$ ), we investigated individual differences regarding preference for index-finger or whole-hand pointing. Sixty-three percent of the index-finger pointers pointed more often with the index finger than with the whole hand (mean proportion of indexfinger points $=.83$ ), and $37 \%$ of the index-finger pointers revealed the reverse pattern and pointed more often with the whole hand than the index finger (mean proportion of index-finger points $=.23$ ). This difference within the index-finger pointers was statistically not significant (binomial test, $p=.359$ ) and not related to the overall frequency of pointing or any other measures reported in this section, thus justifying formation of groups based on simple presence or absence of index-finger pointing.

With regard to vocalizations, the majority of infants (78\%) accompanied their pointing at least once with a vocalization (with a mean proportion of .64); 35\% accompanied their pointing at least once with a "da" (with a mean proportion of .40). Index-finger pointing was related to point-accompanying vocalizations. Fig. 3 shows that index-finger pointers displayed a significantly greater proportion of point-accompanying vocalizations than hand pointers (Mann-Whitney $U=85.5 ; p=.008$ ), and the proportional use of the index finger and the proportion of point-accompanying vocalizations were significantly correlated (Spearman's $\rho=.338, p=.041$ ). Thus, infants who point with the index finger point more frequently and their pointing is more likely to be accompanied by vocalizations.

\subsection{Mothers' pointing}

The decorated room elicited spontaneous, uninstructed pointing in all but 3 mothers. The distribution ranged from 0 to 30 points with a median of 8 and a mean of 8.85. All points were with the index finger and accompanied by speech.

Table 1 shows that significantly more infants who pointed above the median had mothers who pointed above the median, $p h i=.332, p=.038$. Mothers' and infants' absolute numbers of points were not significantly related to each other, suggesting no such direct link as mimicking or mirrored activation. Further analyses revealed that the relation between mothers' and infants' pointing 


Vocalizing 确 "Da!" $\square$ No Vocalizing

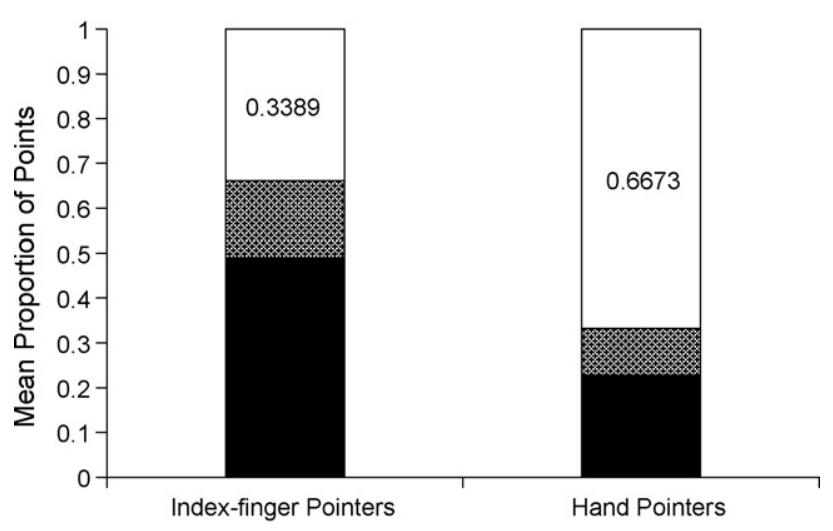

Fig. 3. Proportion of point-accompanying vocalizations in index-finger pointers and hand pointers.

\section{Table 1}

Number of infants and mothers who pointed above their median point distributions.

\begin{tabular}{llr}
\hline Number of infants pointing & \multicolumn{2}{l}{ Number of mothers pointing } \\
\cline { 2 - 3 } & >median & $\leq$ median \\
\hline >median & 12 & 6 \\
$\leq$ median & 7 & 14 \\
\hline
\end{tabular}

was influenced by infants' pointing forms. Fig. 4 shows that index-finger pointers pointed overall significantly more than their mothers, while other infants tended to point less than their mothers (respectively, Wilcoxon's $Z=2.53, p=.011$; Wilcoxon's $Z=1.85, p=.061$ ).

Infants and mothers thus pointed spontaneously in a situation which afforded looking at things together, supporting the hypothesis that pointing is used as a practice of looking together at objects. In

Infants $\square$ Caregivers

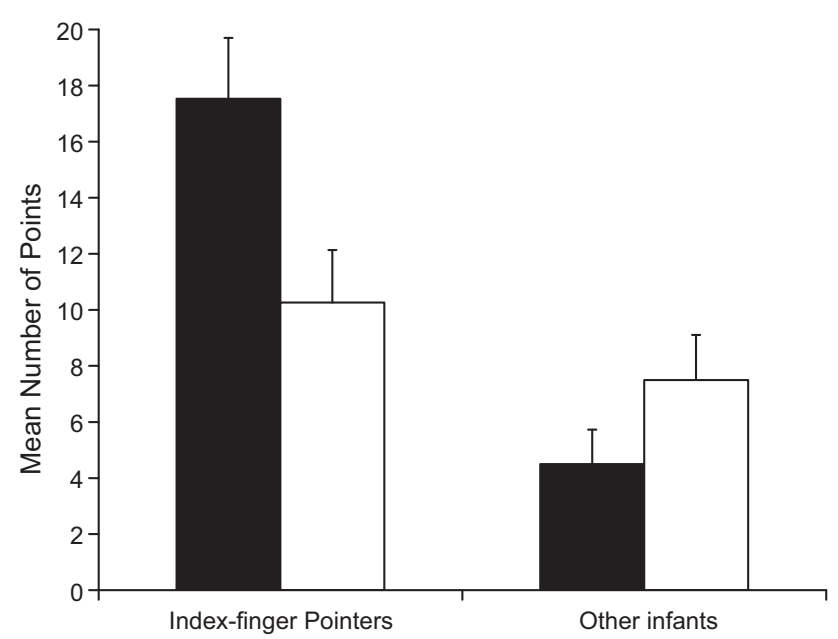

Fig. 4. Mean number of points for index-finger pointers and other infants and their mothers. 


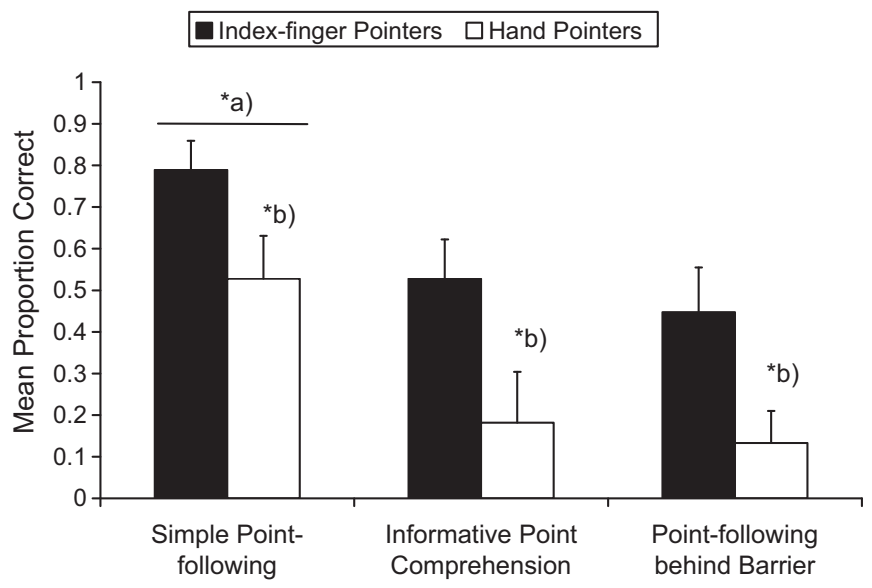

Fig. 5. Performance in the three point comprehension tests for index-finger pointers and hand pointers. (*a) Significant difference relative to other two tasks; ( $\left.{ }^{*} b\right)$ significant differences between index-finger and hand pointers.

addition, mothers who pointed a lot had infants who pointed a lot, supporting the social-interactional hypothesis that infant pointing is a shared social practice from the beginning. Finally, index-finger pointing and whole-hand pointing differed in a number of ways. The pointing of index-finger pointers was better integrated with the vocal modality than that of infants who pointed only with the whole hand. Further, whereas whole-hand pointing was stable in frequency across the two groups and coexisted with index-finger pointing, the group of index-finger pointers pointed more frequently than the group of hand pointers. Based on these individual differences, one developmental implication is that once infants point with the index finger their usage is significantly different from other, presumably earlier forms of whole-hand pointing both qualitatively and quantitatively.

\subsection{Relations to point comprehension, imitation, and object permanence assessments}

Preliminary analyses revealed no differences between index-finger pointers $(n=19)$ and other infants with regard to age, parental education, family status, birth order, or gender, and these variables did not relate to any of the measures reported in this section. Since some index-finger pointers pointed more often with the whole hand than with the index finger, and others the reverse, we also compared these two groups. As reported earlier, no differences appeared, validating formation of groups based on simple presence or absence of index-finger pointing.

\subsubsection{Point comprehension}

Simple point following. Of all 39 infants, $77 \%$ followed pointing to the lateral target at least once, with a mean proportion of .63 trials. The proportion of point-following correlated significantly with both the number of whole-hand points (Spearman's $\rho=.355, p=.026$ ) and index-finger points (Spearman's $\rho=.345, p=.032$ ). Fig. 5 shows that index-finger pointers followed pointing more often than hand pointers (Mann-Whitney $U=114 ; p=.055$ ).

At the individual level, infants who point-followed were significantly more often index-finger pointers ( $p h i=.358, p=.029$ ). There were significantly more hand pointers who point-followed $(n=12)$ than index-finger pointers who did not point-follow $(n=1)$, McNemar test, $p=.003$. Infants' pointfollowing did not correlate significantly with mothers' pointing.

Informative point comprehension. Eight infants were fussy after the demonstration and had to be excluded from the analyses. Of the 31 remaining infants, $52 \%$ searched for the train in the target location at least once. Point comprehension was related to the number of index finger but not to whole-hand points (respectively, Spearman $\rho=.407, p=.023$; Spearman $\rho=.041, p=.825$ ). Fig. 5 shows that indexfinger pointers performed significantly better than hand pointers (Mann-Whitney $U=52.5 ; p=.024$ ), 


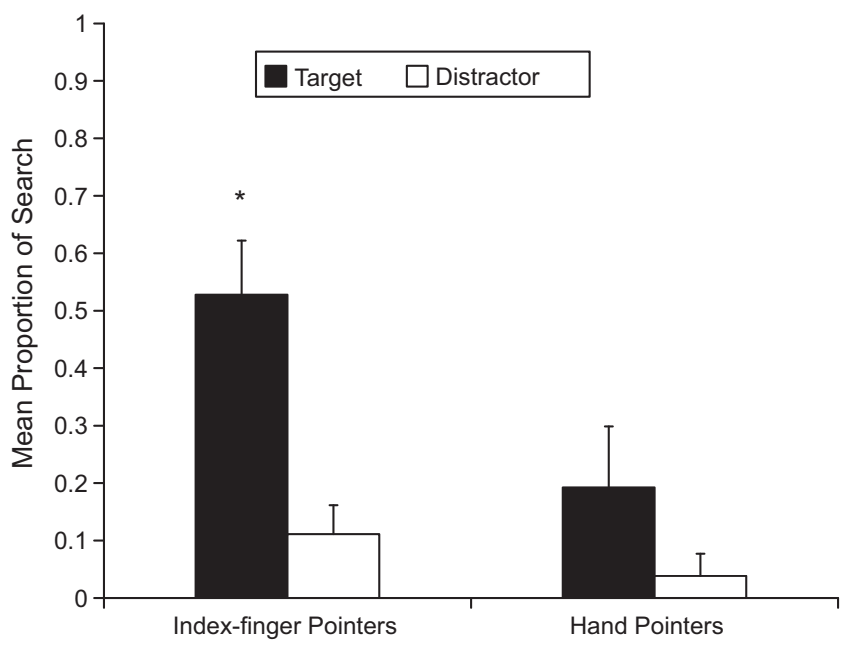

Fig. 6. Correct searches in the informative point comprehension test for index-finger pointers and hand pointers.

and Fig. 6 shows that index-finger pointers - but not hand pointers - searched significantly more often in the target than distractor location (Wilcoxon's $Z=2.76, p=.006$ ).

At the individual level, infants who searched correctly at least once were significantly more often index-finger pointers ( $\mathrm{phi}=.525, p=.005$ ). Five index-finger pointers did not search correctly, compared to two hand pointers who did search correctly (McNemar test, $p=.453$ ). Infants' correct searching did not correlate significantly with mothers' pointing.

Point-following behind barrier. Four infants were fussy during the trials and had to be excluded from the analyses. Of the 35 remaining infants, $37 \%$ followed pointing behind the barrier at least once, with a mean proportion of 30 trials. This proportion is comparable to 12-month-olds' performance in a similar test of gaze-following behind a barrier (40\% of infants reported by Moll \& Tomasello, 2004, Exp. 1). Point comprehension was related to index-finger but not to whole-hand points (respectively, Spearman $\rho=.415, p=.013 ; \rho=-.036, p=.836$ ). Fig. 5 shows that index-finger pointers followed pointing behind the barrier more often than hand pointers (Mann-Whitney $U=90.5 ; p=.037$ ).

At the individual level, infants who point-followed behind the barrier were more often index-finger pointers ( $p h i=.333, p=.051$ ). Nine index-finger pointers did not point-follow compared to three hand pointers who did point-follow (McNemar test, $p=.146$ ). Infants' point following behind the barrier also correlated significantly with mothers' pointing (Spearman's $\rho=.356, p=.036$ ).

Inter-task relations. There was a positive relation between informative point comprehension and the point-following behind barrier tests for both the number of infants who passed and the proportion of trials correct, respectively, $p h i=.455, p=.014$; Spearman's $\rho=.419, p=.024$. Simple point-following did not correlate with either of the two tasks (Spearman's $\rho=.130, .180 ; p=.485, .302$, respectively). Fig. 5 shows that infants performed significantly better in the simple point-following test compared to both the informative point comprehension test and the point-following behind barrier test (respectively, Wilcoxon's $Z=2.8, p=.005$; Wilcoxon's $Z=3.46, p=.001$ ), with no significant differences between the latter two tests.

\subsubsection{Imitation}

Object-directed imitation. In the dumbbell test, 2 infants were fussy and had to be excluded. Of the 37 remaining infants, 73\% copied the action goal and pulled off the cap after E's demonstration (mean proportion of .64 trials). In this task, the goal was more salient than the style and only a few infants (20\%) copied the action style (mean proportion of .19 trials). In the bird test, this pattern was the reverse, with the action style being more salient than the action goal. Of 35 infants, 52\% copied the action style (mean proportion of .35 trials) and hopped the bird around, and $29 \%$ copied the action 
goal (mean proportion of .24 trials). Subsequently, we analyzed imitation of style and goal within and across the two tests with regard to differences in, and relations to infants' pointing status, number of points, morphology, and mothers' number of points. None of these analyses yielded any significant differences or relations.

Gesture imitation. Only 3 of 30 infants copied one of E's gestures once.

Role reversal. Two infants were fussy and had to be excluded. In the first demonstration trial, 62\% of infants copied the action goal. As in the object-directed imitation tests, the number of infants who imitated the goal in the first demonstration trial was not related to any of the measures of infants' and mothers' pointing. Only one infant reversed roles at test. This result is different from Carpenter et al.'s (2005) study in which $19 \%$ of the infants reversed roles in a similar test.

Inter-task relations. There were no relations among any of the imitation tests.

\subsubsection{Object permanence}

One infant was fussy and was excluded. Of the remaining 38 infants, $37 \%$ retrieved the toy after it had been hidden with the cup, with a mean proportion of .24 trials. In the Carpenter et al. (1998) study, $20 \%$ passed at 11 months and $58 \%$ at 12 months. Index-finger pointers passed significantly more

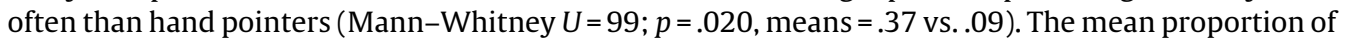
trials with a pass were also positively related to the number of mothers' points (Spearman's $\rho=.376$, $p=.020$ ), such that mothers of infants who passed pointed significantly more than mothers of infants who did not pass (Mann-Whitney $U=91.5 ; p=.019$, means $=12.1$ vs. 6.7 ).

Inter-task relations. There were no relations across tests other than the relation between the informative point comprehension and the point-following behind barrier tests noted previously.

\section{Discussion}

Analyses of performance on tests of point comprehension, imitation, and object permanence produced three main results. First, index-finger pointers performed better than other infants in all three point comprehension tests. Second, for index-finger pointers, but not for other infants, point production and point comprehension were positively correlated in several different ways. Third, simple point-following was significantly easier than the two comprehension tests involving more complex inferences about referential intentions, and only these latter two tests intercorrelated. These results suggest that index-finger pointing involves an understanding of communicative intentions in a way that whole-hand pointing may not.

Imitation was not related to point production or any comprehension measures. In two cases the lack of correlations was due to floor effects. First, in the gesture imitation test, infants did not reproduce gestures without objects. This suggests that twelve-month-olds do not simply mimic others' motor movements outside goal-directed situations. It is also possible that infants became too tired toward the end of the session since the task was administered last, but we did not find any indication of this in their overall behavior. Second, in the role reversal test infants did not reverse roles in their imitations. Although this result is different from Carpenter et al.'s (2005) finding, their study revealed only a low success rate, which may suggest that passing this test is not a precondition for indexfinger pointing. However, looking-time studies suggest that already 10 -month-olds are sensitive to the complementary roles in joint activities (Schöppner, Sodian, \& Pauen, 2006). The object-directed imitation tests yielded enough variation in performance but still failed to produce correlations with pointing. Also the reproduction of an action style - which could be important for copying the specific form of index-finger pointing - was not related to pointing. Other research has suggested that a relation between pointing and imitation is a later development (Camaioni et al., 2004), with communicative gestures emerging before imitative learning skills (Carpenter et al., 1998). The present results suggest that imitation is not the main mechanism in the emergence of pointing. This conclusion is in line with our first analysis revealing that parents modeled points exclusively with the index finger while many infants pointed with the whole hand, although they are perfectly able to extend the isolated index finger from a very early age (Hannan \& Fogel, 1987).

The object permanence test yielded only moderate success rates, and it correlated with infants' point production. However, the success rate in our test was rather low compared to a similar test 
reported by Carpenter et al. (1998), and their study did not find any correlations with deictic gestures and object permanence. This result thus calls for further research. It is possible that our test posed additional communicative demands since it involved a strong communicative component when $\mathrm{E}$ excitedly addressed infants: "Where is the [X]? Hmm? Where is it?" and expectantly looked at the infant, raising her arms, palms up, which is similar to a search procedure in a communicative paradigm (Liszkowski, Carpenter, et al., 2008; Liszkowski, Albrecht, et al., 2008).

Most broadly, a major finding of the present study is that infants' index-finger pointing has a profile completely different from whole-hand pointing. Infants who used the extended index finger pointed more frequently and coordinated their pointing more with the vocal modality. Moreover, infants who pointed with the index finger also comprehended the referential intention behind a point. In addition, infants' pointing was related to their mothers' pointing. These results provide empirical support for social-pragmatic accounts of early pointing, which emphasize both social-interactional and social-cognitive processes, specifically in the case of index-finger pointing.

With regard to the morphology of pointing, our results suggest that index-finger pointing is a separate, presumably later development. Since all index-finger pointers also pointed with the whole hand, it seems unlikely that some infants proceed directly to index-finger pointing without being able to point with the whole hand, while the reverse is certainly the case. Longitudinal studies are needed to confirm this prediction. It is currently unclear whether whole-hand pointing follows a more individualistic or a more social emergence trajectory, but results show that it is used in social contexts and related to simple point-following. Our findings, based on individual differences, thus suggest that (a) the pointing gesture is preceded by some behavioral precursor forms and does not emerge fullblown; and (b) once infants point with the index finger their motor act of reference is integrated with the vocal modality and they point in a full-fledged sense. This interpretation is supported by results with respect to infants' social interactional and social-cognitive skills.

First, with regard to social interaction, results support Werner and Kaplan's (1963) idea that pointing emerges as a shared practice of looking at things together. Interestingly, index-finger pointers exceeded their mothers' pointing. Thus, infants who point with the index finger take the lead in initiating interactions and go already beyond pointing in supported interaction frames. This finding demonstrates that infants, who have just begun pointing fully, actively co-construct with their caregivers social interactional experiences. In contrast, hand pointers pointed less than their mothers. One possibility is thus that parents' pointing provides a minimally leading input and social scaffold in response to infants' emerging whole-hand pointing (Lock et al., 1990). But the current results could also indicate that parents follow their children's lead in pointing. The impact of parents' pointing on the emergence of infants' pointing needs further investigation in longitudinal designs.

Second, with regard to social cognition, results provide evidence of index-finger pointing as a fully communicative act of reference. The two comprehension tests that required inferences about a nonvisible referent both correlated with index-finger pointing - but not with whole-hand pointing. These correlations were also present at the individual level, in particular for the informative point comprehension test, indicating that infants who point with the index finger comprehend pointing in terms of the underlying referential intentions. Point comprehension involves presumably different levels of complexity (Aureli et al., 2009; Liebal \& Tomasello, 2009). Simple point-following only requires orienting toward a single identifiable, visible object, but not necessarily to infer others' referential intentions. It was easier than the other two comprehension tests and did not correlate with them. Further, unlike the other two comprehension tests, simple point-following was present at the individual level among most infants, even if they did not point with the index finger. A longitudinal design is needed to confirm that simple point-following emerges before index-finger pointing and before the comprehension of reference to occluded entities. The fact that simple point-following, unlike the other two comprehension tests, correlated with whole-hand pointing may further suggest that whole-hand pointing already involves some bi-directional understanding of indicating in the sense of turning-for-looking at objects (Werner \& Kaplan, 1963), but not necessarily in the sense of its underlying referential intention. The tendency of index-finger pointers to perform better on the simple point-following test may then reflect a developmental transition from understanding pointing as embodied turning-for-looking to understanding and using pointing as a fully communicative act of reference (Leung \& Rheingold, 1981). 
In support of social-pragmatic accounts, the present study reveals that infants' early pointing is related to social interactional practices of looking together at objects and to social-cognitive skills for comprehending reference. Index-finger pointing is different in this respect from earlier forms of motor reference. The presumably earlier morphological form (mostly whole-hand pointing) is initially more an embodied act of turning-for-looking than it is a fully referential act. It is not well integrated with the vocal modality, it is used relatively infrequently, and it does not involve a robust understanding of referential and social intentions. In any case, once infants point with the index finger, pointing is more frequent, exceeds parental input, is accompanied by communicative vocalizations, and is underlain by a social-cognitive understanding of referential and social intentions. Future studies are needed to disentangle the individual contributions of social-cognitive processing skills and socialinteractional experiences in the emergence of particular forms of pointing. Some researchers take evidence of infant social-cognitive skills and significant task inter-correlations to reveal a cognitive foundation of interaction (Tomasello et al., 2007; Csibra \& Gergely, 2009), while others take the absence of inter-correlations among some cognitive tasks to indicate a social-relational emergence of social understanding (Carpendale \& Lewis, 2004; Carpendale \& Carpendale, 2010). Current results suggest that the ontogenetic origins of reference lie in both the shared social practice and the cognitive skills of looking at things together, which turn infants' initially embodied looking and indicating into fullfledged communicative acts with a bi-directional understanding of reference once infants point with the index finger around 12 months of age.

\section{Acknowledgements}

We thank Amy Whitehouse for her extensive help with the study set-up and data collection, Malinda Carpenter for comments on an earlier draft of the manuscript, and Marie Schäfer and Anja Gampe for help with coding.

\section{References}

Aureli, T., Perucchini, P., \& Genco, M. (2009). Children's understanding of communicative intentions in the middle of the second year of life. Cognitive Development, 24, 1-12.

Bates, E., Camaioni, L., \& Volterra, V. (1975). The acquisition of performatives prior to speech. Merrill-Palmer Quarterly, 21, 205-226.

Bates, E., Dale, P., \& Thal, D. (1995). Individual differences and their implications for theories of language development. In P. Fletcher, \& B. MacWhinney (Eds.), Handbook of child language (pp. 96-151). Oxford: Basil Blackwell.

Baron-Cohen, S., Cox, A., Baird, G., Swettenham, J., Drew, A., Nightingale, N., et al. (1996). Psychological markers of autism at 18 months of age in a large population. British Journal of Psychiatry, 168, 158-163.

Behne, T., Carpenter, M., \& Tomasello, M. (2005). One-year-olds comprehend the communicative intentions behind gestures in a hiding game. Developmental Science, 8, 492-499.

Bruner, J. (1983). Child's talk. New York: Norton.

Butterworth, G. (2003). Pointing is the royal road to language for babies. In S. Kita (Ed.), Pointing: Where language, culture, and cognition meet (pp. 9-33). Mahwah, NJ, USA: Lawrence Erlbaum Associates, Publishers.

Camaioni, L., Perucchini, P., Bellagamba, F., \& Colonnesi, C. (2004). The role of declarative pointing in developing a theory of mind. Infancy, 5, 291-308.

Capirci, O., Iverson, J. M., Pizzuto, E., \& Volterra, V. (1996). Communicative gestures during the transition to two-word speech. Journal of Child Language, 23, 645-673.

Carpendale, J. I. M., \& Carpendale, A. B. (2010). The Development of Pointing: From Personal Directedness to Interpersonal Direction. Human Development, 53, 110-126.

Carpendale, J. E. M., \& Lewis, C. (2004). Constructing an understanding of mind: The development of children's understanding of mind within social interaction. Behavioral and Brain Sciences, 27, 79-150.

Carpenter, M., Nagell, K., \& Tomasello, M. (1998). Social cognition, joint attention, and communicative competence from 9 to 15 months of age. Monographs of the Society of Research in Child Development, 63(4) [Serial No. 176].

Carpenter, M., Tomasello, M., \& Striano, T. (2005). Role reversal imitation and language in typically-developing infants and children with autism. Infancy, 8, 253-278.

Csibra, G., \& Gergely, G. (2009). Natural pedagogy. Trends in Cognitive Sciences, 13, 148-153.

Delgado, B., Gómez, J. C., \& Sarriá, E. (2009). Private pointing and private speech: Developing parallelisms. In A. Winsler, C. Fernyhough, \& I. Montero (Eds.), Private speech, executive functioning, and the development of verbal self-regulation. CUP.

Franco, F., \& Butterworth, G. (1996). Pointing and social awareness: Declaring and requesting in the second year. Journal of Child Language, 23, 307-336.

Franco, F., Perucchini, P., \& March, B. (2009). Is infant initiation of joint attention by pointing affected by type of interaction? Social Development, 18, 51-76.

Hannan, T., \& Fogel, A. (1987). A case-study assessment of "pointing" during the first three months of life. Perceptual and Motor Skills, 65, 187-194. 
Harris, M., Barlow-Brown, F., \& Chasin, J. (1995). The emergence of referential understanding: Pointing and the comprehension of object names. First Language, 15, 19-34.

Iverson, J. M., \& Goldin-Meadow, S. (2005). Gesture paves the way for language development. Psychological Science, 16, $367-371$. Lempers, J. D. (1979). Young children's production and comprehension of nonverbal deictic behaviors. Journal of Genetic Psychology, 135, 93-102.

Leung, \& Rheingold. (1981). Development of pointing as a social gesture. Developmental Psychology, 17, 215-220.

Liebal, K., \& Tomasello, M. (2009). Infants appreciate the social intention behind a pointing gesture: Commentary on “Children's understanding of communicative intentions in the middle of the second year of life" by T. Aureli, P. Perucchini and M. Genco. Cognitive Development, 24, 13-15.

Liszkowski, U., Albrecht, K., Carpenter, M., \& Tomasello, M. (2008). Infants' visual and auditory communication when a partner is or is not visually attending. Infant Behavior and Development, 31, 157-167.

Liszkowski, U., Carpenter, M., \& Tomasello, M. (2007). Reference and attitude in infant pointing. Journal of Child Language, 34(1), $1-20$.

Liszkowski, U., Carpenter, M., \& Tomasello, M. (2008). Twelve-month-olds communicate helpfully and appropriately for knowledgeable and ignorant partners. Cognition, 108, 732-739.

Lock, A., Young, A., Service, V., \& Chandler, P. (1990). Some observations on the origins of the pointing gesture. In V. Volterra, \& C. J. Erting (Eds.), From gesture to language in hearing and deaf children (pp. 42-55). Berlin: Springer-Verlag.

Masataka, N. (2003). From index-finger extension to index-finger pointing: Ontogenesis of pointing in preverbal infants. In S. Kita (Ed.), Pointing: Where language, culture, and cognition meet (pp. 69-84). Mahwah, NJ, USA: Lawrence Erlbaum Associates, Publishers.

Meltzoff, A. N. (1995). Understanding the intentions of others: Re-enactment of intended acts by 18-month-old children. Developmental Psychology, 31, 1-16.

Moll, H., \& Tomasello, M. (2004). Twelve- and 18-month-old infants follow gaze to spaces behind barriers. Developmental Science, 7, F1-F9.

Moore, C., \& Corkum, V. (1994). Social understanding at the end of the first year of life. Developmental Review, 14, 349-372.

Mundy, P., Block, J., Delgado, C., Pomares, Y., Vaughan Van Hecke, A., \& Parlade, M. V. (2007). Individual differences and the development of joint attention in infancy. Child Development, 78, 938-954.

Murphy, C. M., \& Messer, D. J. (1977). Mothers, infants and pointing: A study of a gesture. In H. R. Schaffer (Ed.), Studies in mother-infant interaction. New York: Academic.

Rheingold, H., Hay, D., \& West, M. (1976). Sharing in the second year of life. Child Development, 47, 1148-1158.

Schöppner, B., Sodian, B., \& Pauen, S. (2006). Encoding action roles in meaningful social interaction in the first year of life. Infancy, 9, 289-311.

Tomasello, M., Carpenter, M., \& Liszkowski, U. (2007). A new look at infant pointing. Child Development, 78, 705-722.

Uzgiris, I. C., \& Hunt, J. (1975). Assessment in infancy: Ordinal scales of psychological development. Urbana, IL: University of Illinois Press.

Vygotsky. (1978). Mind in society: The development of higher psychological processes. Cambridge, MA: Harvard University Press.

Werner, H., \& Kaplan, B. (1963). Symbol formation: An organismic-developmental approach to language and the expression of thought. New York: Wiley.

Wilkins, D. (2003). Why pointing with the index finger is not a universal. In S. Kita (Ed.), Pointing: Where language, culture, and cognition meet (pp. 171-215). Mahwah, NJ: Lawrence Erlbaum Associates. 